\title{
PHASE DIAGRAM OF THE SELFINTERACTING PARTICLE-ANTIPARTICLE BOSON SYSTEM
}

\author{
D. Anchishkin ${ }^{1 *}$, V. Gnatovskyy ${ }^{2}$, D. Zhuravel ${ }^{1}$, V. Karpenko ${ }^{2}$ \\ ${ }^{1}$ Bogolyubov Institute for Theoretical Physics, National Academy of Sciences of Ukraine, 03143 Kyiv, \\ Ukraine \\ ${ }^{2}$ Taras Shevchenko National University of Kyiv, 03022 Kyiv, Ukraine \\ *e-mail:dmanch@bitp.kiev.ua
}

\begin{abstract}
A system of interacting relativistic bosons at finite temperatures and isospin densities is studied within the framework of the Skyrme-like mean-field model. The mean field contains both attractive and repulsive terms. The consideration is taken within the framework of the Canonical Ensemble and the isospin-density dependencies of thermodynamic quantities is obtained, in particular as the phase diagrams. It is shown that in such a system, in addition to the formation of a Bose-Einstein condensate, a liquid-gas phase transition is possible. We prove that the multi-boson system develops the Bose condensate for particles of high-density component only.

Keywords: relativistic bosonic system, Bose-Einstein condensation, strong interaction.
\end{abstract}

Received 23.07.2021; Received in revised form 11.08.2021; Accepted 15.08.2021

\section{Introduction}

It is commonly accepted that QCD exhibits a rich phase structure at finite temperatures and baryon densities, for instance, the transition process from hadron gas to quark-gluon plasma, the transition from chiral symmetry breaking to the symmetry restoration [1]. Recently, the study on the QCD phase structure is extended to finite isospin density [2-7]. The physical motivation to study QCD at finite isospin density and the corresponding pion system is related to the investigation of compact stars, isospin asymmetric nuclear matter and heavy ion collisions. In early studies on dense nuclear matter and compact stars, it has been suggested that charged pions and even kaons are condensed at sufficiently high densities. Interesting new results concerning dense pion systems have been obtained recently using lattice methods [8-10]. Hence, the knowledge of the phase structure of the meson systems, in the regime of finite temperatures and isospin densities is crucial for understanding of a wide range of phenomena from nucleus-nucleus collisions to boson, neutron stars and cosmology. This field is an essential part of investigations of hot and dense hadronic matter which is a subject of active research. Meanwhile, investigations of the meson systems has its own specifics due to a possibility of the Bose-Einstein condensation (BEC) of interacting bosonic particles.

In the present paper we consider an interacting particle-antiparticle boson system at the conserved isospin (charge) density $n_{I}$ and finite temperature $T$. We name the bosonic particles as "pions" just conventionally. The preference is made because the charged $\pi$-mesons are the lightest hadrons that couple to the isospin chemical potential. On the other hand, the pions are the lightest nuclear boson particles and thus, an account for "temperature creation" of particleantiparticle pairs is a relevant problem on the basis of quantum statistics. To account for the interaction between the bosons we introduce a phenomenological Skyrme-like mean field $U(n)$, which depends only on the total meson density $n$. We regard such a selfinteracting many-particle system, as a toy-model that can help us understand the BEC and phase transitions over a wide range of temperatures and densities. The mean field $U(n)$ rather reflects the presence of other strongly interacting particles in the system, for instance $\rho$-mesons and nucleon-antinucleon pairs at low temperatures or gluons and quark-antiquark pairs at high temperatures, $T>T_{\text {qgp }} \approx$ $160 \mathrm{MeV}$.

The presented study is a part of a sequel which is started from investigation of an interacting 
particle-antiparticle boson system at $\mu=0$ [11-14]. Next, a development of the subject was given in refs. $[15,16]$, where the same boson system was considered within the framework of the Canonical Ensemble with the canonical variables $T, n_{I}$, i.e. the temperature and isospin density. In this formulation we calculated the temperature characteristics of a non-ideal hot "pion" gas with a fixed isospin density $n_{I}=n^{(-)}-n^{(+)}>0$, where $n^{(\mp)}$ are the particlenumber densities of the $\pi^{-}$and $\pi^{+}$mesons, respectively. In the present study we proceed to exploit the Canonical Ensemble, but now we are focusing on the isospin-density dependencies of thermodynamic quantities when the temperature is fixed.

\section{The mean-field model for the system of boson particles and antiparticles}

We consider a particle-antiparticle system of bosons with the isospin number $n_{I}=n^{(-)}-$ $n^{(+)}$, where $n^{(-)}$is the particle-number density of bosonic particles and $n^{(+)}$is the particlenumber density of bosonic antiparticles. The chemical potentials of boson particles $\mu_{\mathrm{p}}$ and boson antiparticles $\mu_{\mathrm{p}}$ have opposite signs:

$$
\mu_{\mathrm{p}}=-\mu_{\mathrm{p}} \equiv \mu .
$$

Therefore, the Euler relation includes isospin number density only:

$$
\varepsilon+p=T s+\mu n_{I} .
$$

Here $\varepsilon, p$ and $s$ are the energy density, pressure and entropy density, respectively. The total particle-number density is $n=n^{(-)}+n^{(+)}$.

Thermodynamic mean field model (see details in [17]) introduces two quantities which depend on the total particle-number density $n$ : mean field $U(n)$ and excess pressure $P(n)$. Using these quantities the total pressure in the two-component particle-antiparticle system can be written as ${ }^{1}$

$$
\begin{aligned}
p= & -g T \int \frac{d^{3} k}{(2 \pi)^{3}} \ln \left[1-\exp \left(-\frac{\sqrt{m^{2}+\mathbf{k}^{2}}+U(n)-\mu}{T}\right)\right]- \\
& -g T \int \frac{d^{3} k}{(2 \pi)^{3}} \ln \left[1-\exp \left(-\frac{\sqrt{m^{2}+\mathbf{k}^{2}}+U(n)+\mu}{T}\right)\right]+P(n),
\end{aligned}
$$

where $g$ is the degenerate factor.

The thermodynamic consistency of the mean-field model can be obtained by putting in correspondence of two expressions which must coincide in the result. These expressions, which determine the isospin density, read

$$
n_{I}=\left(\frac{\partial p}{\partial \mu}\right)_{T}, \quad \text { and } \quad n_{I}=g \int \frac{d^{3} k}{(2 \pi)^{3}}[f(E(k, n), \mu)-f(E(k, n),-\mu)],
$$

where pressure is given by eq.(3). Here $E(k, n)=\omega_{k}+U(n)$ with $\omega_{k}=\sqrt{m^{2}+\mathbf{k}^{2}}$ and the Bose-Einstein distribution function reads

$$
f(E, \mu)=\left[\exp \left(\frac{E-\mu}{T}\right)-1\right]^{-1} .
$$

\footnotetext{
${ }^{1}$ Here and below we adopt the system of units $\bar{h}=c=1, k_{B}=1$
} 
In order the expressions (4) to coincide in the result the following relation between the mean field and the excess pressure arises

$$
n \frac{\partial U(n)}{\partial n}=\frac{\partial P(n)}{\partial n} .
$$

It provides the thermodynamic consistency of the model.

\subsection{Skyrme-like parametrization of the mean field}

The thermodynamic mean-field model has been applied for several physically interesting systems including the hadron-resonance gas [17] and the pionic gas [20]. In the present study this formalism is used to describe two-component (particle-antiparticle) system of pions. We assume that the interaction between particles is described by the Skyrme-like mean field which depends only on the total particle-number density $n$. Loosely speaking we take into account just a strong interaction. So, in the framework of the proposed toy-model we adopt that the mean field has the following form

$$
U(n)=-A n+B n^{2},
$$

where $A$ and $B$ are the model parameters, which should be specified. As was mentioned in the introduction, an investigation of the properties of a dense and hot pion gas is well inspired by formation of the medium with low baryon numbers at midrapidity what was proved in the experiments at RHIC and LHC [21,22].

By this reason, in our calculations we consider a general case of $A \geq 0, B \geq 0$, to study a bosonic system with both attractive and repulsive contributions to the mean field (7). For the repulsive coefficient $B$ we use a fixed value, obtained from an estimate based on the virial expansion [23], $B=10 m v_{0}^{2}$ with $v_{0}$ equal to four times the proper volume of a particle, i.e. $v_{0}=16 \pi r_{0}^{3} / 3$. In our numerical calculations we take $v_{0}=0.45 \mathrm{fm}^{3}$ that corresponds to a "particle radius" $r_{0} \approx 0.3 \mathrm{fm}$. The numerical calculations will be done for bosons with mass $m=139 \mathrm{MeV}$, which we call "pions". In this case the repulsive coefficient is $B / m=2.025 \mathrm{fm}^{6}$ and it is kept constant through all present calculations. At the same time the coefficient $A$, which determines the intensity of attraction of the mean field (7), will be varied. It is advisable to parameterize the coefficient $A$ in the following way: let us use solutions of equation $U(n)+m=$ 0 , a similar algorithm was adopted in refs. [11,13]. For the given mean field (7) there are two roots of this equation

$$
n_{1}=\sqrt{\frac{m}{B}}\left(\kappa-\sqrt{\kappa^{2}-1}\right), \quad n_{2}=\sqrt{\frac{m}{B}}\left(\kappa+\sqrt{\kappa^{2}-1}\right),
$$

where

$$
\kappa \equiv \frac{A}{2 \sqrt{m B}} .
$$

Then, one can parametrize the attraction coefficient as $A=\kappa A_{\mathrm{c}}$ with $A_{\mathrm{c}}=2 \sqrt{m B}$. As we will show below, the dimensionless parameter $\kappa$ is the scale parameter of the model, it determines the phase structure of the system. We consider two intervals of the parameter $\kappa$. First interval corresponds to $\kappa \leq 1$, as it is seen from eq. (8) there are no real roots. We associate these values of $\kappa$ with a "weak" attractive interaction and in the present study we consider variations in the attraction coefficient $A$ for values of $\kappa$ only from this interval. Second interval corresponds to $\kappa>1$, there are two real roots. We associate this interval with a "strong" attractive interac- 
tion. This case will be considered elsewhere. The critical value $A_{\mathrm{c}}$ is obtained when both roots coincide, i.e. when $\kappa=1$, then $A=A_{\mathrm{c}}=2 \sqrt{m B}$.

We are going now to consider the basic thermodynamic states of the bosonic system using the mean field (7).

\section{Isospin dependence of thermodynamic properties of the boson system}

In the mean-field approach the behavior of the particle-antiparticle bosonic system in thermal (kinetic) phase is determined by the set of two transcendental equations (see eq. (4))

$$
\begin{aligned}
n & =n^{(-)}+n^{(+)}=\int \frac{d^{3} k}{(2 \pi)^{3}}\left[f_{\mathrm{BE}}(E(k, n), \mu)+f_{\mathrm{BE}}(E(k, n),-\mu)\right], \\
n_{I} & =n^{(-)}-n^{(+)}=\int \frac{d^{3} k}{(2 \pi)^{3}}\left[f_{\mathrm{BE}}(E(k, n), \mu)-f_{\mathrm{BE}}(E(k, n),-\mu)\right],
\end{aligned}
$$

where $g=1$, the Bose-Einstein distribution function $f_{\mathrm{BE}}(E, \mu)$ is defined in (5) and $E(k, n)=$ $\omega_{k}+U(n)$. Equations (10)-(11) should be solved self-consistently with respect to $n$ and $\mu$ for a given temperature $T$ and isospin density $n_{I}$. In the present we consider boson system in the Canonical Ensemble, where the independent canonical variables are $T$ and $n_{I}$, and the spin of particles equals to zero. In this approach the chemical potential $\mu$ is a thermodynamic quantity which depends on the canonical variables, i.e. $\mu\left(T, n_{I}\right)$.

As was shown in Refs. $[15,16]$ for the "weak" interaction, $\kappa \leq 1$, in the temperature interval $T \leq T_{\mathrm{c}}$ the particles, i.e. $\pi^{-}$-mesons, are in the condensate phase and antiparticles, i.e. $\pi^{+}$-mesons, are in the thermal (kinetic) phase. It was obtained that the low-density component in the two-component particle-antiparticle system, in the present case it is $\pi^{+}$-mesons, does not develop the condensate phase at all, i.e. the antiparticles are in thermal phase for $0 \leq T<\infty$. That is why, eqs. (10), (11) which describe both components being in thermal phase should be generalized to include condensate contribution $n_{\text {cond }}^{(-)}$to high-density component of the pion gas in the interval $T \leq T_{\mathrm{c}}$. We should also take into account that the particles, i.e. $\pi^{-}$, can develop the condensate states just under the necessary condition

$$
U(n)-\mu=-m .
$$

During decreasing of temperature from high values, where both $\pi^{-}$and $\pi^{+}$mesons were in thermal phase, the density of $\pi^{-}$-component $n^{(-)}\left(T, n_{I}\right)$ achieves the critical curve at temperature $T_{\mathrm{c}}$, where condition (12) is valid. Under condition (12) the particle-number density $n^{(-)}$, see eq. (10), transforms to $n^{(-)}=n_{\lim }(T)$, where $n_{\lim }(T)$ is defined as

$$
n_{\lim }(T)=\left.\int \frac{d^{3} k}{(2 \pi)^{3}} f_{\mathrm{BE}}\left(\omega_{k}, \mu\right)\right|_{\mu=m} .
$$

This is the critical curve for $\pi^{-}$-mesons or for high-density component. As we see, when $\mu=m$ the function (13) represents the maximal density of thermal (kinetic) particles of the ideal boson gas at temperature $T$ and it plays the role of the critical curve. Hence, we obtain that in the mean-field approach for boson system with the conserved isospin (charge) density the critical curve coincides with the critical curve for the single-component ideal gas. 
With account for eqs. (12) and (13) we write the generalization of the set of eqs. (10), (11)

$$
\begin{aligned}
n & =n_{\text {cond }}^{(-)}(T)+n_{\lim }(T)+\left.\int \frac{d^{3} k}{(2 \pi)^{3}} f_{\mathrm{BE}}(E(k, n),-\mu)\right|_{\mu=U(n)+m}, \\
n_{I} & =n_{\text {cond }}^{(-)}(T)+n_{\lim }(T)-\left.\int \frac{d^{3} k}{(2 \pi)^{3}} f_{\mathrm{BE}}(E(k, n),-\mu)\right|_{\mu=U(n)+m} .
\end{aligned}
$$

In the presence of condensate, i.e. in the interval $T \leq T_{\mathrm{c}}$, the pressure is calculated as

$$
p=\frac{1}{3} \int \frac{d^{3} k}{(2 \pi)^{3}} \frac{\mathbf{k}^{2}}{\omega_{k}}\left[f_{\mathrm{BE}}\left(\omega_{k}, m\right)+\left.f_{\mathrm{BE}}(E(k, n),-\mu)\right|_{\mu=U(n)+m}\right]+P(n),
$$

where the particle-number density $n$ includes the condensate contribution.
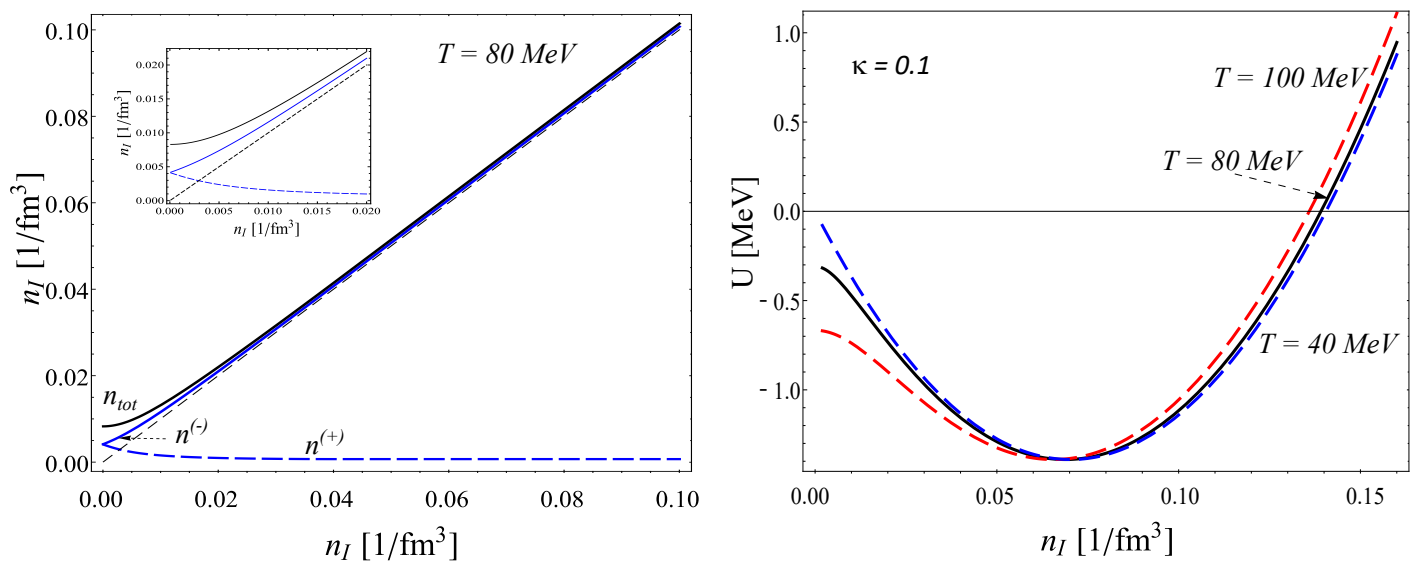

Fig. 1. Left panel: The particle-number densities $n^{(+)}, n^{(-)}$and $n_{\text {tot }}=n^{(+)}+n^{(-)}$versus isospin density $n_{I}$ at $T=80 \mathrm{MeV}, \kappa=0.1$ for the interacting $\pi^{+}-\pi^{-}$pion gas in the mean-field model. The black dashed straight line is a linear dependence $n=n_{I}$. Right panel: The mean field $U(n)$ versus isospin density $n_{I}$ at $\kappa=0.1$ and $T=40,80,100 \mathrm{MeV}$ in the interacting $\pi^{+}-\pi^{-}$pion gas.

\subsection{Numerical results}

We are going to analyze the phase structure of the particle-antiparticle system with respect to the canonical variable $n_{I}$. On this way it is instructive to obtain firstly the dependencies of $n^{(-)}, n^{(+)}$and $U$ with respect to $n_{I}$ when we fix $T$. Every isotherm crosses two different phases, thus for every particular phase we have to solve the relevant set of equations: 1) when both components of the pion gas are in thermal (kinetic) states one solves eqs. (10), (11); 2) when $\pi^{-}$-component of the pion gas has a condensate contribution one solves eqs. (14), (15). For the chosen isotherm $T$ the point $n_{I}=n_{\text {Ic }}$ divides $n_{I}$-axis into two pieces. When $n_{I} \leq n_{\text {Ic }} \pi^{-}$- and $\pi^{+}$-mesons are in thermal phase. While for $n_{I}>n_{\mathrm{Ic}} \pi^{-}$-mesons have condensate contribution and $\pi^{+}$-mesons are in thermal phase. Along this way the particle-number densities $n^{(+)}, n^{(-)}$ and $n_{\text {tot }}=n^{(+)}+n^{(-)}$were calculated as functions of isospin density $n_{I}$ at $T=80 \mathrm{MeV}$, $\kappa=0.1$. Results are depicted in Fig. 1 on left panel. One can see that for $n_{I} \gtrsim 0.03 \mathrm{fm}^{-3}$ the density of $\pi^{-}$mesons virtually coincide with the total particle-number density $n$ (in figure notations we use $n_{\text {tot }}$ ). It is seen also that the density of $\pi^{+}$mesons decreases with increase of $n_{I}$. In fact, this behavior is quite understood: when $n_{I}>n_{\mathrm{Ic}}$ an increase of the total density $n$ is going on just due to an increase of the condensate contribution of $n^{(-)}$, thermal contribution of $\pi^{-}$mesons, $n_{\mathrm{th}}^{(-)}$, has a maximum value for the fixed temperature $T$ when $n_{I}>n_{\mathrm{Ic}}$. At the 
same time the density $n^{(+)}$slightly decreases.

The dependence of the mean field on $n_{I}$ for three values of temperature is shown in Fig. 1 on the right panel. It is seen that after the minimum of $U\left(n\left(T, n_{I}\right)\right)$ with increasing $n_{I}$, the difference in the curves associated with temperature is very weak. In the point $n_{I}=n_{\mathrm{I} 0}$, where $U\left(n_{\mathrm{I} 0}\right)=0$, the mean field changes its sign and becomes completely repulsive, at $\kappa=0.1$ we obtained $n_{\mathrm{I} 0} \approx 0.14 \mathrm{fm}^{-3}$.
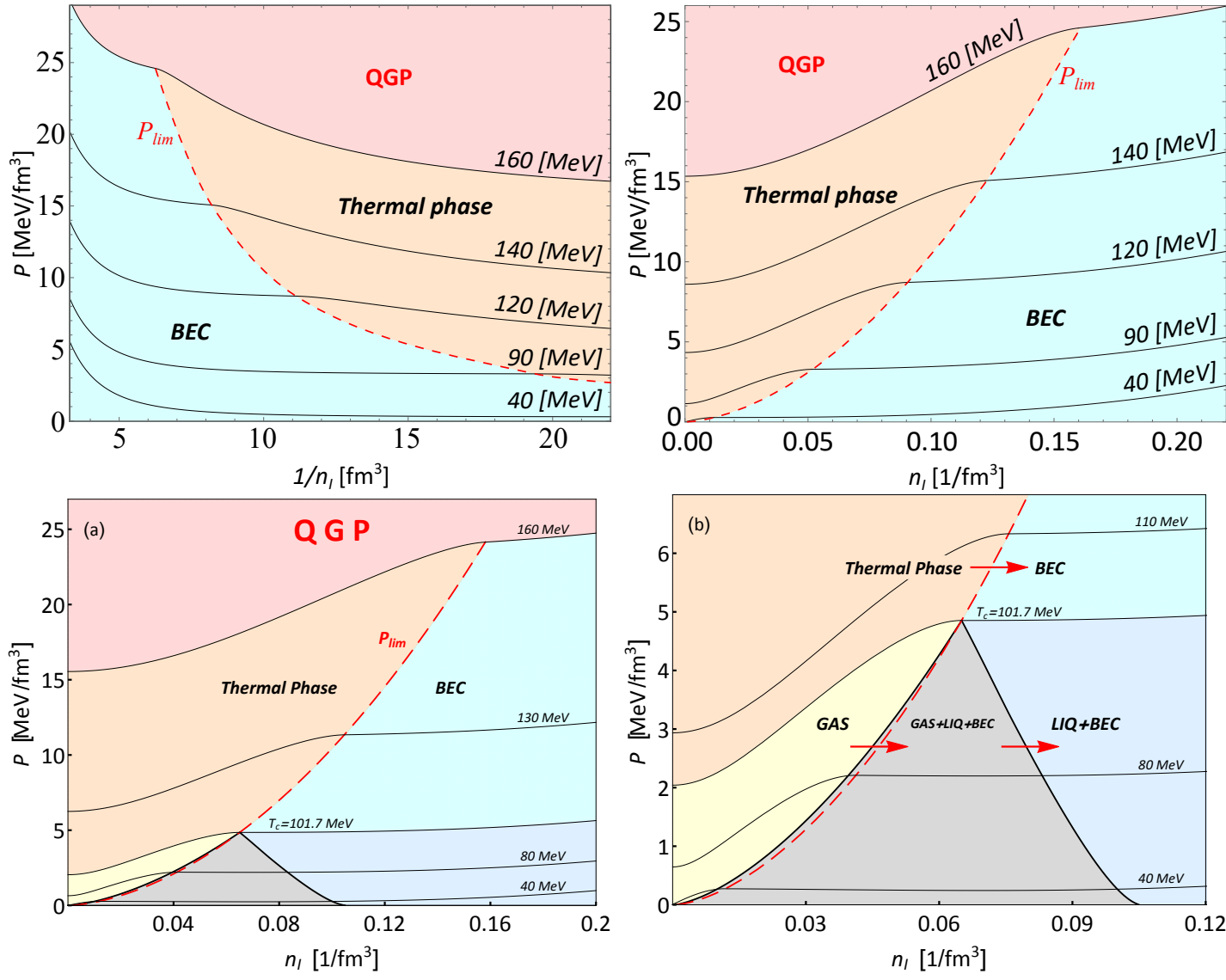

Fig. 2. Phase diagrams. The isotherm $T=160 \mathrm{MeV}$ is the limit of the QGP phase. The red dashed curve $p_{\text {lim }}$ separates thermal phase from the BEC phase. Upper row. Pressure versus inverse isospin density $v=1 / n_{I}$ (left panel) and versus isospin density $n_{I}$ (right panel) in the interacting $\pi^{-}-\pi^{+}$gas with repulsion between particles, $\kappa=0$. Lower row. Panel (a):Isotherms in the interacting $\pi^{+}{ }_{-}^{-}$pion gas at $\kappa=0.1$. Below the critical isotherm $T_{\mathrm{c}}=101.7 \mathrm{MeV}$ the liquid-gas phase transition takes place. Panel (b): The same as in panel (a) but for greater scale.

With the aim to analyze the phase structure of the particle-antiparticle system we look for a dependence of pressure $p\left(T, n_{I}\right)$ on $n_{I}$ when the temperature is fixed. It means that we investigate the behavior of pressure on the isotherm $T$ with respect to the canonical variable $n_{I}$. At the first step the system with only repulsion between particles, i.e. $\kappa=0$, was tested. The phase structure of the system in this case is depicted on two upper panels in Fig. 2 for the dependencies $p=p\left(v_{I}\right)$ and $p=p\left(n_{I}\right)$, where $v_{I}=1 / n_{I}$. In every panel we see three different phases: 1) "Thermal phase" - particles and antiparticles are both in thermal states; 2) "BEC" the subsystem of particles have the Bose-Einstein condensate contribution and the subsystem of antiparticles, $\pi^{+}$-mesons, is in thermal phase; 3) "QGP" - the phase where the quark-gluon plasma occurs, this phase is separated by the isotherm $T=T_{\text {qgp }}=160 \mathrm{MeV}$ (we assume a 
melting of all pion states at temperatures $T>T_{\mathrm{qgp}}$ ). The line $p_{\text {lim }}$ is the pressure of $\pi^{-}$mesons on the critical curve $n_{\text {lim }}$. As it is evidently seen the behavior of isotherms is slightly changes after crossing the the curve $p_{\text {lim }}$.

After "switching on" the attraction between particles the isotherms from the interval $T<$ $T_{\mathrm{c}}$ exhibit a "sine-like" behavior in finite interval $\Delta n_{I}$. In accordance with the standard thermodynamic approach this specific behavior of the isotherms can be treated as a liquid-gas phase transition. In accordance with that we apply the Maxwell rules and obtain the binodal, which determines the region of the liquid-gas phase transition in a similar way as was done in refs. $[18,19]$. The resulting phase diagram is shown in Fig. 2 like the two lower panels, which differ from each other only in scale. The mixed liquid-gas phase (shaded gray area) appears to be almost entirely in the condensate phase (labeled GAS+LIQ+BEC on the right panel). As it follows from the Maxwell rules the pressure associated with the isotherms which cross the mixed liquid-gas phase has a constant value. We would like to draw attention once more that the density of condensate in the two-component pion system is created by $\pi^{-}$-mesons only, i.e. $n_{\text {cond }}=n_{\text {cond }}^{(-)}$. That is why, the particle-number density $n_{\text {cond }}$ plays the role of the order parameter.

\subsection{Bulk modulus}

The bulk modulus $K$ of a substance characterizes how the pressure varies during compression at a constant-temperature. It is defined as the ratio of the infinitesimal pressure increase to the relative decrease of the volume of the system and in the Canonical Ensemble it reads

$$
K \equiv-V\left[\frac{\partial p\left(T, N_{I}, V\right)}{\partial V}\right]_{T}
$$

Meanwhile, because the volume is inversely proportional to the total particle-number density $n$ one can rewrite expression (17) in terms of the particle-number density as

$$
K=n\left[\frac{\partial p\left(T, n_{I}\right)}{\partial n}\right]_{T}=n\left(T, n_{I}\right) \frac{\left[\frac{\partial p\left(T, n_{I}\right)}{\partial n_{I}}\right]_{T}}{\left[\frac{\partial n\left(T, n_{I}\right)}{\partial n_{I}}\right]_{T}},
$$

or

$$
K=n_{I}\left[\frac{\partial p\left(T, n_{I}\right)}{\partial n_{I}}\right]_{T} .
$$

If the total particle-number density depends on spatial variables, then the bulk modulus depends also on spatial variables and thus reflects the spatial properties of the many-particle system. Results of calculation of the bulk modulus in accordance with eq. (18) at $\kappa=0$ for different temperatures is depicted in Fig. 3 on the upper panels. As can be seen when $\kappa=0$ the bulk modulus experience a break in a point of the BEC transition. When we "switch on" attraction in the system the bulk modulus becomes zero in the region, where the liquid-gas phase transition takes place, i.e. for the isotherms $T<T_{\mathrm{c}}$. This effect is depicted on the lower panels in Fig. 3.

\section{Concluding remarks}

This article presents a thermodynamically consistent method for describing a dense bosonic system of interacting $\pi^{-}$-mesons and $\pi^{+}$-mesons. We considered the system of meson particles with $m=m_{\pi}$ and zero spin, because the charged $\pi$-mesons are the lightest nuclear particle and the lightest hadrons that couple to the isospin number. Description of thermodynamic proper- 

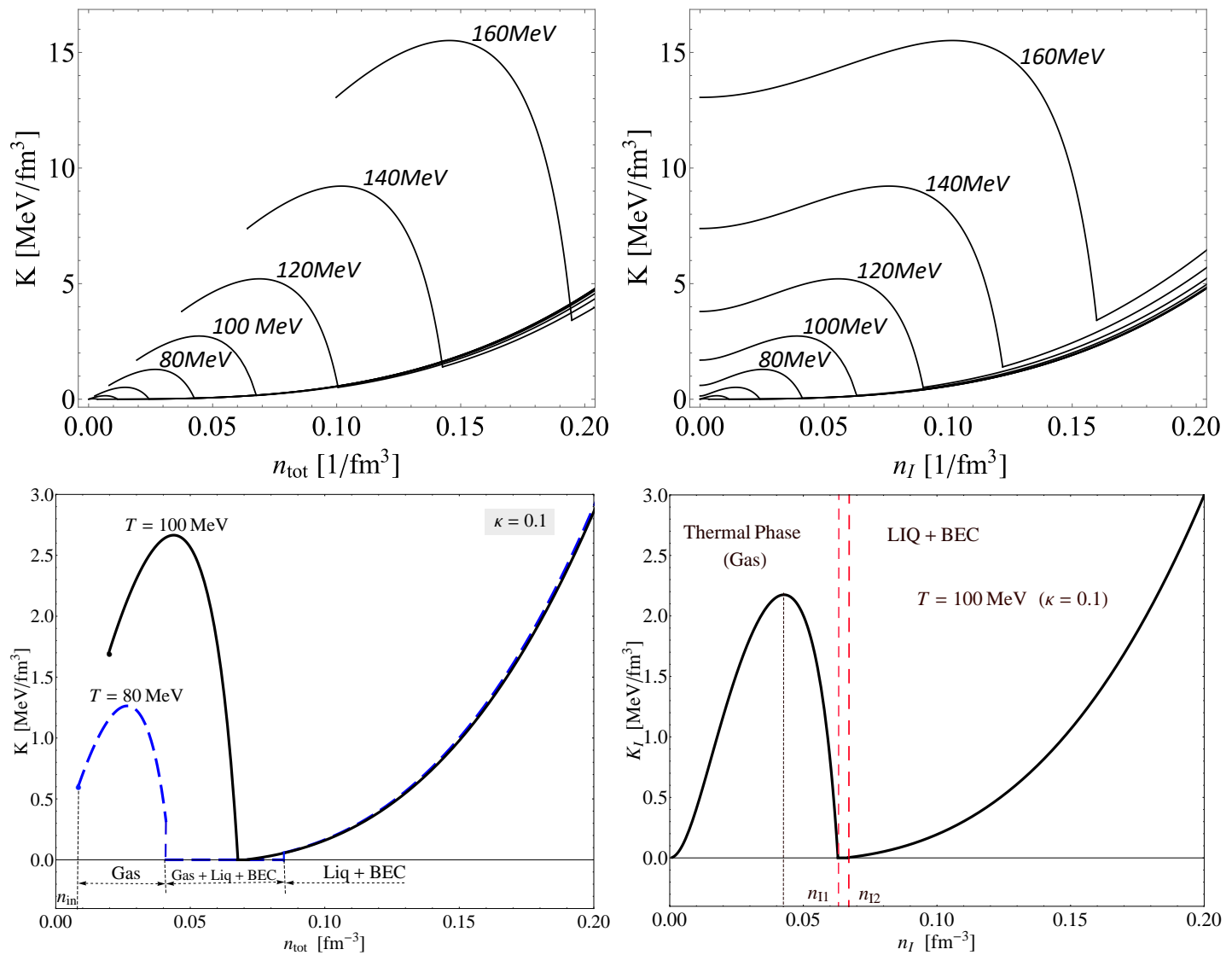

Fig. 3. Upper panels: Bulk modulus versus the total particle density $n_{\text {tot }}=n$ (left panel) and versus the isospin density (right panel) in the pion gas with repulsion between all particles, $\kappa=0$. Lower panels: Bulk modulus versus total particle-number density (left panel) at $T=80,100 \mathrm{MeV}, \kappa=0.1$ (see eq. (18)). Bulk modulus versus isospin density (right panel) at $T=100 \mathrm{MeV}, \kappa=0.1$ (see eq. (19)). The Liquid+Gas+BEC phase is in between the red dashed vertical lines where pressure is constant and thus $K=0$.

ties of the system was performed employing the Canonical Ensemble formulation, where the chemical potential $\mu$ is a thermodynamic quantity which depends on the canonical variables $\left(T, n_{I}\right)$. To obtain phase diagrams we calculated dependence of pressure on the isospin density for different values of $T$.

It was shown that $n^{(-)}\left(n_{I}\right)$ and $n_{\text {tot }}\left(n_{I}\right)$ dependencies with respect to $n_{I}$ are almost linear and close to one another for $n_{I}>n_{I c}$ since for every fixed $T$ the value of thermal-particle density $n_{\text {th }}^{(-)}$does not change [16] and the value of $n^{(+)}$is approximately constant (see Fig. 1). Because only $\pi^{-}$mesons undergo the phase transition to the Bose-Einstein condensate, the increase of the densities $n^{(-)}$and $n_{t o t}$ for $n_{I}>n_{I c}$ is due to increase of the density of condensate.

Phase diagrams were introduced on Fig. 2. The scale parameter of the model $\kappa=A /(2 \sqrt{m B})$, which is itself a combination of the mean-field parameters $A$ and $B\left(U(n)=-A n+B n^{2}\right)$ and the particle mass, determines the different possible phase scenarios which occur in the particleantiparticle boson system. When attraction coefficient $A=\kappa A_{c}$, where $A_{c} \equiv 2 \sqrt{m B}$, is equal to zero (i.e. $\kappa=0$ ), the system can be only in the thermal phase or in the condensate phase. In the case of $\kappa>0$, liquid-gas phase transition occurs in the system, and a transition from the thermal phase to the condensate one is possible both with liquid-gas phase transition if $T<T_{\mathrm{c}}$, and without it when $T \geq T_{\mathrm{c}}$. In other words there is a region in the phase diagram where the BEC and the mixed liquid-gas phase exist simultaneously (grey area on the lower panels in Fig. 2). A 
similar situation is described in [19], where the Bose-Einstein condensation and the liquid-gas phase transition in $\alpha$-matter were investigated. Area above isotherm $T=T_{\mathrm{qgp}}=160 \mathrm{MeV}$ (QGP) is the phase where the quark-gluon plasma occurs. We assume this to be a limitation of our model since a melting of all pion states at temperatures higher than $T_{\text {qgp }}$.

In the section 3.2, we introduced the calculation of the bulk modulus of the system of interaction pions. As can be seen on the upper panels in Fig. 3 when $\kappa=0$ the bulk modulus experience a break in a point of the BEC transition. As soon as we "turn on" attraction $(\kappa>0$, lower panels) and the liquid-gas transition occurs, the bulk modulus turns to zero in this region. The reason for that is that the pressure is constant due to the Maxwell rules. In first case $(\kappa=0)$, the special behavior of the bulk modulus can be used to determine the BEC transition in the system with repulsion between particles. In second case $(\kappa>0)$, the drop of the bulk modulus to zero gives a signal about the presence of a liquid-gas phase transition in the bosonic system.

\section{Acknowledgements}

The work of D. A. is supported by the National Academy of Sciences of Ukraine by its priority project "Fundamental properties of the matter in the relativistic collisions of nuclei and in the early Universe" (No. 0120U100935). The work of D. Zh. is supported by the Program "The structure and dynamics of statistical and quantum-field systems" of the Department of Physics and Astronomy of NAS of Ukraine.

\section{References}

1. Bzdak, A. Mapping the Phases of Quantum Chromodynamics with Beam Energy Scan / A. Bzdak, S. Esumi, V. Koch, J. Liao, M. Stephanov, N. Xu // Physics Reports.-2020.- Vol. 853.- P. 1-87.

2. Son, D.T. QCD at Finite Isospin Density / D.T. Son, M.A. Stephanov // Phys. Rev. Lett.2001.- Vol. 86, 592; [arXiv:hep-ph/0005225]; Phys. Atom. Nucl.- 2001.- Vol. 64, 834.

3. Kogut, J. QCD at small non-zero quark chemical potentials / J. Kogut, D. Toublan // Phys. Rev. D.- 2001.- Vol. 64, 034007; arXiv:hep-ph/0103271.

4. Toublan, D. Isospin Chemical Potential and the QCD Phase Diagram at Nonzero Temperature and Baryon Chemical Potential / D. Toublan, J. Kogut // Phys. Lett. B.- 2001.- Vol. 564, 212; arXiv:hep-ph/0301183.

5. Mammarella, A. Intriguing aspects of meson condensation / A. Mammarella, M. Mannarelli // Phys. Rev. D.- 2015.- Vol. 92, 085025; arXiv:1507.02934 [hep-ph].

6. Carignano, S. Scrutinizing the pion condensed phase / S. Carignano, L. Lepori, A. Mammarella, M. Mannarelli, G. Pagliaroli // Eur. Phys. J. A.- 2017.- Vol. 53, 35; arXiv: 1610.06097 [hep-ph].

7. Mannarelli, Massimo Meson condensation / Massimo Mannarelli //Particles.-2019.- Vol. 2, 411; arXiv:1908.02042 [hep-ph].

8. Brandt, B.B. QCD phase diagram with isospin chemical potential / B.B. Brandt, G. Endrödi // PoS LATTICE2016.- 2016.- Vol. 039; arXiv:1611.06758 [hep-lat].

9. Brandt, B.B. QCD at finite isospin chemical potential / B.B. Brandt, G. Endrödi, S. Schmalzbauer // EPJ Web Conf.- 2018.- Vol. 175, 07020; arXiv:1709.10487 [hep-lat].

10. Brandt, B.B. QCD phase diagram for nonzero isospin-asymmetry / B.B. Brandt, G. Endrődi, S. Schmalzbauer // Phys. Rev. D.- 2018.- Vol. 97, 054514; arXiv:1712.08190 [heplat].

11. Anchishkin, D. Phase Transition in Interacting Boson System at Finite Temperatures/ D. Anchishkin, I. Mishustin, H. Stoecker // J. Phys. G.- 2019.-Vol. 46, No.3, 035002; 
arXiv:1806.10857 [nucl-th].

12. Mishustin, I.N. Condensation of interacting scalar bosons at finite temperatures / I.N. Mishustin, D.V. Anchishkin, L.M. Satarov, O.S. Stashko, H. Stoecker // Phys. Rev. C.2019.- Vol. 100, 022201(R); arXiv:1905.09567 [nucl-th].

13. Anchishkin, D. Finite-Temperature Bose-Einstein Condensation in Interacting Boson System / D. Anchishkin, I. Mishustin, O. Stashko, D. Zhuravel, H. Stoecker // Ukranian J. Phys.- 2019.- Vol. 64, No. 12.- P. 1110-1116.

14. Stashko, O.S. Thermodynamic properties of interacting bosons with zero chemical potential / O.S. Stashko, D.V. Anchishkin, O.V. Savchuk, M.I. Gorenstein // J. Phys. G: Nucl. Part. Phys.-2020, DOI: 10.1088/1361-6471/abd5a5; arXiv: 2007.06321 [hep-ph].

15. Anchishkin, D. Relativistic Selfinterecting Particle-Antiparticle System of Bosons / D. Anchishkin, V. Gnatovskyy, D. Zhuravel, V. Karpenko // Journal of Physics and Electronics.2020.- Vol. 28 (2).-P. 3-18; DOI 10.15421/332016

16. Anchishkin, D. Selfinteracting Particle-Antiparticle System of Bosons / D. Anchishkin, V. Gnatovskyy, D. Zhuravel, V. Karpenko // arXiv:2102.02529 [hep-ph].

17. Anchishkin, D. Mean-field approach in the multi-component gas of interacting particles applied to relativistic heavy-ion collisions / D. Anchishkin, V. Vovchenko // J. Phys. G: Nucl. Part. Phys.- 2015.- Vol. 42, 105102.- P. 1-27; arXiv:1411.1444 [nucl-th].

18. Lee, S. J. Nuclear Chemical and Mechanical Instability and the Liquid-Gas Phase Transition in Nuclei / S.J. Lee, A.Z. Mekjian // arXiv:0709.0228v1 [nucl-th].

19. Satarov, L. M. Bose-Einstein condensation and liquid-gas phase transition in $\alpha$-matter / L. M. Satarov, M. I. Gorenstein, A. Motornenko,V. Vovchenko, I.N. Mishustin, H. Stoecker // J. Phys. G: Nucl. Part. Phys.- 2017.- Vol. 44, 125102;arXiv:1704.08039v1 [nucl-th], DOI 10.1088/1361-6471/aa8c5d

20. Poberezhnyuk, R. V. Limiting temperature of pion gas with the van der Waals equation of state / R.V. Poberezhnyk, V.Yu. Vovchenko, D.V. Anchishkin, M.I. Gorenstein // J. Phys. G: Nucl. Part. Phys.- 2016.- Vol. 43, 095105; arXiv:1508.04585 [nucl-th].

21. Adamczyk, L. Bulk properties of the medium produced in relativistic heavy-ion collisions from the beam energy scan program / L. Adamczyk et al. [STAR Collab.] // Phys. Rev. C.2017.- Vol. 96, 044904.

22. Abelev, B. Pion, kaon, and proton production in central $\mathrm{Pb}-\mathrm{Pb}$ collisions at $\sqrt{s_{N N}}=$ 2.76 TeV / B. Abelev et al. [ALICE Collab.] // Phys. Rev. Lett.- 2012. - Vol. 109, 252301.

23. Hansen, J. P. Theory of Simple Liquids / J.P. Hansen, I.R. McDonald // Academic Press. 2006. 